\title{
Primary prevention of gestational diabetes mellitus through nutritional factors: a systematic review
}

\author{
Mikel Donazar-Ezcurra ${ }^{1 *}$, Cristina López-del Burgo ${ }^{1,2,3}$ and Maira Bes-Rastrollo 1,2,4
}

\begin{abstract}
Background: Gestational diabetes mellitus (GDM), defined as any degree of glucose intolerance with onset during pregnancy, is increasing worldwide, mostly because obesity among women of reproductive age is continuously escalating. GDM is associated with adverse maternal and fetal outcomes. The aim of this article was to systematically review literature on the effectiveness of nutritional factors before or during pregnancy to prevent GDM.

Methods: We assessed the primary prevention of GDM through nutritional factors, as diet and supplements. We searched on PubMed, Cochrane Databases and ClinicalTrials.gov from inception to June 2016. Clinical trials and adjusted prospective cohort studies were included.

Results: Eight clinical trials and twenty observational studies assessing the association between dietary factors and primary prevention of GDM were included. Furthermore, six clinical trials and two observational studies related to supplements were also added. Only two nutritional interventions were found to significantly reduce the incidence of GDM, besides the supplements. However, the observational studies showed that a higher adherence to a healthier dietary pattern can prevent the incidence of GDM, especially in high risk population before getting pregnant.
\end{abstract}

Conclusions: The results indicate that there may be some benefits of some nutritional factors to prevent GDM. However, better-designed studies are required to generate higher quality evidence. At the moment, no strong conclusions can be drawn with regard to the best intervention for the prevention of GDM.

Keywords: Gestational Diabetes Mellitus, Primary prevention, Nutritional factors, Diet and dietary supplements

\section{Background}

Gestational diabetes mellitus (GDM), defined as any degree of glucose intolerance with onset during pregnancy, is increasing worldwide. In fact, prevalence has increased by $10-100 \%$ in the last 20 years [1]. More mothers entering pregnancy as obese and of advanced maternal age has contributed to the escalation of GDM cases. Several risk factors are known for GDM; advanced maternal age, obesity, physical inactivity, parity, ethnicity, family history of type type 2 diabetes (T2D), history of macrosomic babies, and a previous history of GDM $[2,3]$.

GDM is associated with adverse maternal and fetal outcomes during pregnancy and long term health [4].

\footnotetext{
* Correspondence: mikeldoez@gmail.com

'Department of Preventive Medicine and Public Health, University of Navarra,

C/Irunlarrea1, 31008 Pamplona, Navarra, Spain

Full list of author information is available at the end of the article
}

In order to prevent these adverse outcomes, such as T2D, it is imperative to understand the hormonal changes and altered glucose metabolism that are associated with the development of GDM during pregnancy [5]. GDM occurs when insulin receptors are not able to respond adequately to control blood sugar levels due to hormones produced in pregnancy, such as human placental lactogen, which impacts susceptible insulin receptors. This in turn causes inappropriately high levels of blood sugar. Due to the similarities in the underlying pathophysiology and risk factors of GDM and T2D, it is probable that the factors that are effective in the prevention of T2D may be successful in the prevention of GDM as well. These factors include dietary pattern, physical activity, a decrease in rates of obesity and gestational weight gain. It is important to identify population factors to reduce the increasing rates of GDM as it 
is doing for T2D. The aim of the researchers was to systematically review literature on clinical trials and prospective cohort studies focusing on the effectiveness of nutritional factors (diet and supplements) before or during pregnancy to prevent GDM.

The study protocol is available as a supplementary file in this article.

\section{Methods}

We undertook this systematic review following a protocol in accordance with the current recommendations of PRISMA and MOOSE guidelines [6, 7].

No ethical approval is required for this research project.

\section{Search strategy}

Articles were researched in the following databases: PubMed, Cochrane Databases and ClinicalTrials.gov from inception to June 2016. The keywords used for the search were: "gestational diabetes", "diet" and "nutrition". Another search was done for the keywords of "supplement" and "gestational diabetes". The search strategy was done independently by two researchers, and both extracted the relevant studies to be included in this systematic review. The disagreement between the researchers was resolved consulting a third researcher in order to reach an accord. We restricted publications to English, French and Spanish languages. All the researchers have an advanced level in these three languages. The selection process of the articles began from reading the titles. After this initial phase, the selected papers were reviewed by reading their abstracts. At that time, these selected studies were separated for further analysis to identify relevant publications, according to the inclusion/exclusion criteria. For the selection of studies, inclusion criteria were adopted such as: clinical trials or adjusted prospective cohort studies, originals, and primary prevention of GDM through nutritional factors. Due to inherent limitations other epidemiological studies were excluded. Exclusion criteria were: presence of diabetes mellitus type 1 or 2 prior to pregnancy, presence of diseases prior to pregnancy requiring dietary treatment, evaluation of diet as a treatment for patients with GDM, only physical activity for prevention of GDM and research on animals. Additional articles were identified from the reference lists of relevant studies and reviews.

\section{Results and discussion}

\section{Study selection}

Our initial research in the aforementioned electronic databases produced 1992 citations. Eighty-five articles were identified as potential articles for further full text review. After the next exclusion process, 28 articles were included and another seven were added from reference lists.
The most important reason for exclusion was that diet or supplements were evaluated as a treatment for GDM, not as a primary prevention. We identified a total of 35 relevant articles (Fig. 1. Legend; Flow-chart of prospective cohort studies and clinical trials included in the systematic review. The search used the following combinations of terms: "diet" or "nutrition" and "gestational diabetes mellitus". A second search was done with the terms "supplement" and "gestational diabetes mellitus". Filters; Human; languages: English, French and Spanish; date: up to June 30, 2016). Twenty-eight were related to diet and primary prevention of GDM, eight were clinical trials (Additional file 1: Table S1) and 20 were prospective cohort studies (Additional file 2: Table S2). There were eight articles related to supplements and primary prevention of GDM, six clinical trials (Additional file 3: Table S3) and two prospective cohort studies (Additional file 4: Table S4). Lastly, there was one article [8] that studied whether dietary and supplemental iron were related to the occurrence of GDM (Additional file 2: Tables S2 and Additional file 4: Table S4).

\section{Quality of the included studies}

Of the 14 clinical trials included, only one was not randomized [9]. All women were pregnant when intervention had begun in the clinical trials. The diagnosis of GDM was based on the Oral Glucose Tolerance Test (OGTT), although the measurements of the OGTT was not the same in all articles, some considered the fasting glucose level abnormal when $\geq 5.3 \mathrm{mmol} / \mathrm{l}$ [9-11], but others consider it to be abnormal when $\geq 4.8 \mathrm{mmol} / \mathrm{l}$ [12], $\geq 5.1 \mathrm{mmol} / \mathrm{l}[13,14]$ or $\geq 5.5 \mathrm{mmol} / \mathrm{l}$ [15]. Furthermore, the glucose level readings at $1 \mathrm{~h}$ and at $2 \mathrm{~h}$ were also

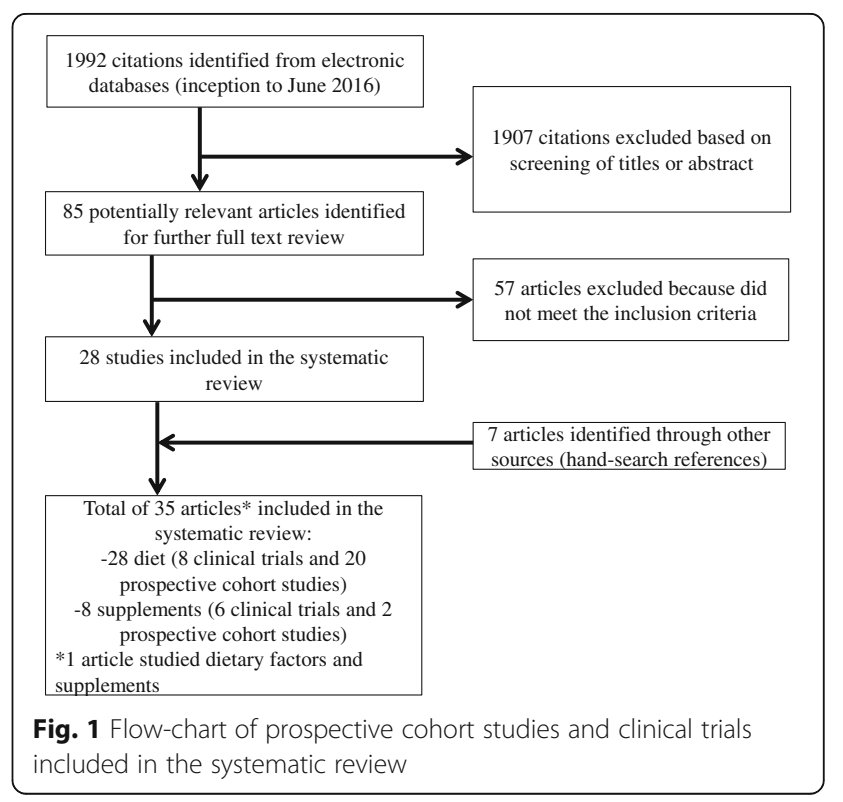


different. One article studied GDM incidence as a secondary outcome [16] and another one does not give a definition of GDM, although they studied glucose blood levels during the pregnancy of the participants [17].

The observational studies were adjusted for the most important variables (such as age, parity, tobacco products, BMI, physical activity, family history of diabetes, alcohol, ethnicity, total calories consumed). Twelve of these articles $[8,18-28]$ were based on the Nurses' Health Study II cohort, which includes more than 13,000 women who reported a singleton pregnancy. In this cohort, GDM cases were identified through self-reported information. The principal measures estimated in these studies were Risk Ratios (RR) and differences in means of GDM incidence.

\section{Main findings}

On one hand, no diet intervention was found to significantly reduce the incidence of GDM except for the intervention of the non-randomized controlled pragmatic trial [11] and the intervention on lifestyle (including diet) of Koivusalo et al. [10], (see Additional file 1: Table S1). Wolff et al. [17] found that an intensive intervention reduces the deterioration of glucose metabolism in obese pregnant women, though they did not report any incidence of GDM. Only two supplement interventions with probiotics and myo-inositol during pregnancy showed a decrease in the rates of GDM compared with a placebo. Luoto et al. [12] intervention showed that probiotics (Lactobacillus Rhamnosus GG and Bifidobacterium Lactis Bb12) reduced the incidence of GDM; 13\% (diet/ probiotics) versus 36\% (diet/placebo) and 34\% (control), $p=0.003$. Luoto et al. [12] explain in their article that probiotic consumption may protect against GDM because these microorganisms can modify intestinal microbiota, altering the fermentation of dietary polysaccharides and improving intestinal barrier function. They also mentioned the importance of the capability of probiotics to regulate the inflammatory pathways. It is understandable that the protection against GDM provided by probiotics, could be mediated through immunomodulatory pathways and polysaccharide fermentation. Moreover, myo-inositol supplements were found to reduce the incidence of GDM in pregnant women (Matarrelli et al. [14]: $R R=0.127$; 95\% CI, 0.032-0.502; $p=0.001$ and D'Anna et al. [13]: $\mathrm{OR}=0.34 ; 95 \% \mathrm{CI}, 0.17-0.68 ; p=0.001)$ and appear to be an insulin sensitizer. It was reported to reduce plasma glucose levels in insulin resistant conditions such as polycystic ovary syndrome and during the third trimester of GDM pregnancies [29]. The subjacent mechanism of myo-inositol on metabolic benefits is not defined at the moment. It may produce an intracellular effect directly on the activation of acetil CoA carboxylase stimulating lipogenesis. Another theory says that it is a precursor of
D-chiro-inositol, which contains inositol phosphoglycan in the extracellular matrix of the cells. It has been proposed that the binding of insulin to specific receptors stimulates D-chiro-inositol, facilitating the transport to the inside of the cell [30]. This explains how myo-inositol interacts in the insulin-signaling cascade [31].

On the other hand, the observational studies collected in Table S2 (Additional file 2) showed that achieving a healthier dietary pattern [25], such a Mediterranean dietary pattern, and lowering the intake of foods with high heme iron content, sugar sweetened cola, potatoes, fatty foods and sweets, can reduce the incidence of GDM, especially among the high-risk population and before getting pregnant $[8,21-23,32-34]$.

Known evidence indicates that women who develop GDM have altered functions of $\beta$-cells and insulin resistance, limiting their capacity to cope with the metabolic challenges of pregnancy [35]. Similarly, it is known that iron is a redox-active transitional metal, a strong prooxidant which promotes the creation of hydroxyl radicals, increasing oxidative stress. The pancreatic $\beta$-cell is particularly sensitive to this type of stress due to weak antioxidant protection [36]. Nevertheless, adherence to healthy diets, such as the Mediterranean one, may reduce GDM risk by minimizing such susceptibilities before pregnancy. Common components of these dietary patterns include fruits and vegetables, relatively small amounts of red and processed meats, and high quality carbohydrates. Fruits and vegetables in particular have many antioxidant properties, in addition to providing fiber and micronutrients such as magnesium and vitamin $\mathrm{C}$. The combination of all these factors may protect against metabolic deterioration counteracting free radicals and improving systemic oxidative stress [37].

\section{Limitations}

It is possible that a publication bias may have occurred as there is a limited amount of publications related to the study of primary intervention of GDM through diet, and absorption of supplements, probiotics and other minerals. In addition, the researchers are not discounting the existence of other articles in databases that were not used for this study.

\section{Clinical applications}

In this systematic review we found that the majority of the interventions done during pregnancy are not effective in preventing GDM, and although there were cases where supplements proved to be beneficial, the evidence is very scarce. However, dietary patterns prior to pregnancy seem to reduce the risk of GDM. Programs encouraging young women to achieve a healthy dietary pattern before getting pregnant seem to be the best way to prevent GDM. Measures adopted during pregnancy seem to be ineffective 
because they require more time to properly curb the development of GDM, especially among the high-risk population.

\section{Implications for future research}

There is a strong need for additional research on this topic. Although some prospective cohort studies have found an association between some nutrients or dietary patterns and the incidence of GDM, the existence of consistency of the results in other populations should be very desirable to prove causal associations between nutritional factors and the risk of GDM. We did not find any controlled trial that evaluated the intervention in dietary pattern before patients get pregnant and the incidence of GDM. Nevertheless, there is an ongoing European multicenter randomized controlled trial in overweight and obese pregnant women on lifestyle intervention and/or vitamin D supplementation [38]. Researchers have recently published the results of the pilot study finding that a healthy eating has a preventive impact on the risk of GDM [39]. Final results of the trial will be published in the near future.

\section{Conclusions}

Overall, since there is evidence that some dietary patterns, such as the Mediterranean diet, seem to lower the risk of developing GDM, and since it has also been shown to prevent other diseases (T2D, metabolic syndrome, cardiovascular diseases), the advice to adhere to this type of dietary pattern could be the best way at present to prevent GDM. Furthermore, it can be a good way to prevent other pregnancy related diseases, such as, hypertension and growth restriction. However, better designed prospective and intervention studies, providing high-quality data are required before conclusions can be drawn about the best intervention for the prevention of GDM.

\section{Additional files}

Additional file 1: Table S1. Characteristics of clinical trials for the primary prevention of Gestational Diabetes Mellitus through dietary factors [9-11, 15-17, 40, 41]. (DOCX $18 \mathrm{~kb})$

Additional file 2: Table S2. Characteristics of prospective cohort studies for the primary prevention of Gestational Diabetes Mellitus through dietary factors [8, 18-28, 32-34, 42-46]. (DOCX 28 kb)

Additional file 3: Table S3. Characteristics of clinical trials for the primary prevention of Gestational Diabetes Mellitus through supplements [12-14, 47-49]. (DOCX $15 \mathrm{~kb})$

Additional file 4: Table S4. Characteristics of prospective cohort studies for the primary prevention of Gestational Diabetes Mellitus through supplements $[8,50]$. (DOCX $13 \mathrm{~kb})$

\section{Abbreviations}

GDM: Gestational diabetes mellitus; OGTT: Oral glucose tolerance test; T2D: Type 2 diabetes

\section{Acknowledgements}

The authors thank the library of the University of Navarra for the cooperation in achieving the data required as well for the technical support

\section{Funding}

CIBERobn Physiopathology of Obesity and Nutrition, Spanish Government Instituto de Salud Carlos III and the European Regional Development Fund (FEDER). Grants: Pl13/00615, PI14/01668, the Navarra Regional Government (122/2014) and the University of Navarra.

\section{Availability of data and materials}

All data generated or analysed during this study are included in this published article.

\section{Authors' contributions}

$M D E, C L B$ and MBR designed research; MDE and MBR conducted the literature search and extracted the data and this was analysed and interpreted by CLB and MBR. MDE drafted the manuscript. This was critically reviewed by $C L B$ and MBR for important intellectual content and subsequent revisions to the paper were undertaken by MDE as a result. All authors approved the final manuscript.

\section{Competing interests}

The authors declare that they have no competing interests.

\section{Consent for publication}

Not applicable.

Ethics approval and consent to participate

Not applicable.

\section{Author details}

'Department of Preventive Medicine and Public Health, University of Navarra, C/Irunlarrea1, 31008 Pamplona, Navarra, Spain. ${ }^{2}$ IDISNA, Navarra's Health Research Institute, Pamplona, Navarra, Spain. ${ }^{3}$ Institute for Culture and Society, University of Navarra, Pamplona, Navarra, Spain. ${ }^{4}$ CIBERobn, Instituto de Salud Carlos III, Madrid, Spain.

Received: 6 May 2016 Accepted: 22 December 2016

Published online: 13 January 2017

References

1. Ferrara A. Increasing prevalence of gestational diabetes mellitus: A public health perspective. Diabetes Care. 2007;30:141-6.

2. Chasan-Taber L, Schmidt MD, Pekow P, et al. Physical Activity and Gestational Diabetes Mellitus among Hispanic Women. J Womens Health. 2008:17:999-1008

3. Teh WT, Teede HJ, Paul E, Harrison CL, Wallace EM, Allan C. Risk factors for gestational diabetes mellitus: Implications for the application of screening guidelines. Aust NZ J Obstet Gynaecol. 2011;51:26-30.

4. Jovanovic L, Pettitt DJ. Gestational Diabetes Mellitus. JAMA. 2001;286:2516-8.

5. Ramírez-Torres MA. The importance of gestational diabetes beyond pregnancy. Nutr Rev. 2013;71:37-41.

6. Moher D, Liberati A, Tetzlaff J. Altman DG and the PRISMA Group. Preferred Reporting Items for Systematic Reviews and Meta-Analyses: The PRISMA Statement Plos Medicine. 2009;6, e1000097.

7. Stroup DF, Berlin JA, Morton SC, et al. Meta-Analysis of Observational studies in Epidemiology. A proposal for reporting. JAMA. 2000;283:2008-12.

8. Bowers $K$, Yeung E, Williams $M$, et al. A prospective study of prepregnancy dietary iron intake and risk for Gestational Diabetes Mellitus. Diabetes Care. 2011;34:1557-63.

9. Luoto R, Kinnunen TI, Aittasalo MI. Primary prevention of gestational diabetes mellitus and large-for-gestational-age newborns by lifestyle counseling: a cluster-randomized controlled trial. PLoS Med. 2011:8, e1001036.

10. Koivusalo SB, Rönö K, Klemetti MM, Roine RP, Lindström J, Erkkola M, et al. Gestational diabetes mellitus can be prevented by lifestyle intervention: the Finnish Gestational Diabetes Prevention Study (RADIEL): a randomized controlled trial. Diabetes Care. 2016;39:24-30.

11. Mustila T, Raitanen J, Keskinen P, Saari A, Luoto R. Pragmatic controlled trial to prevent childhood obesity in maternity and child health care clinics: pregnancy and infant weight outcomes (the VACOPP study). BMC Pediatr. 2013;13:80. 
12. Luoto R, Laitinen $K$, Nermes M, Isolauri E. Impact of maternal probioticsupplemented dietary counselling on pregnancy outcome and prenatal and postnatal growth: a double-blind, placebo-controlled study. Brit J Nutr. 2010;103:1792-9.

13. D'Anna R, Di Benedetto A, Scilipoti A, Santamaria A, Interdonato ML, Petrella

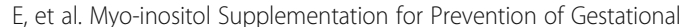
Diabetes in Obese Pregnant Women: A Randomized Controlled Trial. Obstet Gynecol. 2015;126:310-5.

14. Matarrelli B, Vitacolonna E, D'Angelo M, et al. Effect of dietary myo-inositol supplementation in pregnancy on the incidence of maternal gestational diabetes mellitus and fetal outcomes: a randomized controlled trial. J Matern Fetal Neonatal Med. 2013;26:967-72.

15. Markovic TP, Muirhead R, Overs S, Ross GP, Louie JC, Kizirian N, et al. Randomized Controlled Trial investigating the effects of a Low-Glycemic Index Diet on pregnancy outcomes inwomen at high risk of Gestational Diabetes Mellitus: The Gl Baby 3 Study. Diabetes Care. 2016;39:31-8.

16. Asbee SM, Jenkins TR, Butler JR, White J, Elliot M, Rutledge A. Preventing excessive weight gain during pregnancy through dietary and lifestyle Counseling. Obstet Gynecol. 2009;113:305-12.

17. Wolff S, Legarth J, Vangsgaard K, Toubro S, Astrup A. A randomized trial of the effects of dietary counseling on gestational weight gain and glucose metabolism in obese pregnant women. Int J Obes (Lond). 2008; 32:495-501.

18. Bao W, Bowers K, Tobias DK, Hu FB, Zhang C. Prepregnancy dietary protein intake, major dietary protein sources, and the risk of Gestational Diabetes Mellitus. Diabetes Care. 2013:36:2001-8

19. Bao W, Tobias DK, Olsen SF, Zhang C. Pre-pregnancy fried food consumption and the risk of gestational diabetes mellitus: a prospective cohort study. Diabetologia. 2014;57:2485-91.

20. Bao W, Bowers K, Tobias DK, et al. Prepregnancy low-carbohydrate dietary pattern and risk of gestational diabetes mellitus: a prospective cohort study. Am J Clin Nutr. 2014:99:1378-84.

21. Bao W, Tobias DK, Hu FB, Chavarro JE, Zhang C. Pre-pregnancy potato consumption and risk of gestational diabetes mellitus: prospective cohort study. BMJ. 2016;12:352. h6898

22. Bowers $\mathrm{K}$, Tobias DK, Yeung $\mathrm{E}, \mathrm{Hu}$ FB, Zhang C. A prospective study of prepregnancy dietary fat intake and risk of gestational diabetes. Am J Clin Nutr. 2012;95:446-53.

23. Chen L, Hu FB, Yeung E, Willett W, Zhang C. Prospective study of pre-gravid sugar sweetened beverage consumption and the risk of gestational diabetes mellitus. Diabetes Care. 2009;32:2236-41.

24. Chen L, Hu FB, Yeung E, Tobias DK, Willett WC, Zhang C. Prepregnancy consumption of fruits and fruit juices and the risk of Gestational Diabetes Mellitus. Diabetes Care. 2012;35:1079-82.

25. Tobias DK, Zhang C, Chavarro J, et al. Prepregnancy adherence to dietary patterns and lower risk of gestational diabetes mellitus. Am J Clin Nutr. 2012:96:289-95.

26. Zhang C, Schulze MB, Solomon CG, Hu FB. A prospective study of dietary patterns, meat intake and the risk of gestational diabetes mellitus. Diabetologia. 2006;49:2604-13.

27. Zhang C, Liu S, Solomon CG, Hu FB. Dietary fiber intake, dietary glycemic load, and the risk for gestational diabetes mellitus. Diabetes Care. 2006; 29:2223-30

28. Zhang C, Tobias DK, Chavarro JE. Adherence to healthy lifestyle and risk of gestational diabetes mellitus: prospective cohort study. BMJ. 2014;349:95450.

29. Corrado F, D'Anna R, Di Vieste G, et al. The effect of myo-inositol supplementation on insulin resistance in patients with gestational diabetes. Diabet Med. 2011;28:972-5.

30. Baillargeon JP, luorno MJ, Apridonidze T, Nestler JE. Uncoupling between insulin and release of a D-chiro-inositol-containing inositolphosphoglycan mediator of insulin action in obese women with polycystic ovary syndrome. Metab Syndr Relat Disord. 2010;8:127-36.

31. Saltier AR. Second messenger of insulin action. Diabetes Care. 1990;13:244-56

32. He JR, Yuan MY, Chen NN, et al. Maternal dietary patterns and gestational diabetes mellitus: a large prospective cohort study in China. Brit J Nutr. 2015:113:1292-300.

33. Qiu C, Frederick 1O, Zhang C, Sorensen TK, Enquobahrie DA, Williams MA. Risk of gestational diabetes mellitus in relation to maternal egg and cholesterol intake. Am J Epidemiol. 2011;173:649-58.
34. Qiu C, Zhang C, Gelaye B, Enquobahrie DA, Frederick IO, Williams MA. Gestational diabetes mellitus in relation to maternal dietary heme iron and non heme iron intake. Diabetes Care. 2011;34:1564-9.

35. Buchanan TA. Pancreatic B-cell defects in gestational diabetes: implications for the pathogenesis and prevention of type 2 diabetes. J Clin Endocrinol Metab. 2001;86:989-93.

36. Buchanan TA, Xiang A, Kjos SL, Watanabe R. What is gestational diabetes? Diabetes Care. 2007;30:105-11.

37. Hamer M, Chida Y. Intake of fruit, vegetables, and antioxidants and risk of type 2 diabetes: systematic review and meta-analysis. J Hypertens. 2007; 25:2361-9.

38. Jelsma JG, Van Poppel MN, Galjaard S, et al. DALL: Vitamin D and lifestyle intervention for gestational diabetes mellitus (GDM) prevention: an European multicentre, randomised trial-study protocol. BMC Pregnancy Childbirth. 2013;13:142.

39. Simmons D, Jelsma JGM, Galjaard S, Devlieger R, Assche A, Jans G. Results from a European multicenter randomized trial of physical activity and/or healthy eating to reduce the risk of Gestational Diabetes Mellitus: The DALI Lifestyle Pilot. Diabetes Care. 2015;38:1650-6.

40. Thornton YS, Smarkola C, Kopacz SM, Ishoof SB. Perinatal outcomes in nutritionally monitored obese pregnant women: A randomized clinical trial. J Natl Med Assoc. 2009:101:569-71.

41. Vinter CA, Jensen DM, Ovesen P, Bech-Nielsen $H$, Jorgensen JS. TheLiP (LifestyleinPregnancy) Study. A randomized controlled trial of lifestyle intervention in 360 obese pregnant women. Diabetes Care. 2011;34:2502-7.

42. Karamanos B, Thanopoulou A, Anastasiou E, et al. Relation of the Mediterranean diet with the incidence of gestational diabetes. Eur J Clin Nutr. 2014;68:8-13.

43. Radesky JS, Okena E, Rifas-Shimana SL, Kleinmana KP, RichEdwards JW, Gillmana M. Diet during early pregnancy and development of gestational diabetes. Paediatr Perinat Epidemiol. 2008:22:47-59.

44. Saldana TM, Siega-Riz AM, Adair LS. Effect of macronutrient intake on the development of glucose intolerance during pregnancy. Am J Clin Nutr. 2004;79:479-86

45. Wang $Y$, Storlien $L H$, Jenkins $A B$. Dietary variables and glucose tolerance in pregnancy. Diabetes Care. 2000;23:460-4.

46. Zhang C, Williams MA, Sorensen TK. Maternal plasma ascorbic acid (vitamin C) and risk of gestational diabetes mellitus. Epidemiology. 2004;15:597-604.

47. Chan K, Chan B, Lam K, Tam S, Lao T. Iron supplement in pregnancy and development of gestational diabetes-a randomised placebo-controlled trial. BJOG. 2009;116:789-98.

48. Kinnunen TI, Luoto R, Helin A, Hemminki E. Supplemental iron intake and the risk of glucose intolerance in pregnancy: re-analysis of a randomised controlled trial in Finland. Matern Child Nutr. 2014 [epub ahead of print].

49. Zhou SJ, Yelland L, McPhee AJ, Quinlivan J, Gibson RA, Makride M. Fish-oi supplementation in pregnancy does not reduce the risk of gestational diabetes or preeclampsia. Am J Clin Nutr. 2012;95:1378-84.

50. Helin A, Kinnunen TI, Raitanen J, et al. Iron intake, haemoglobin and risk of gestational diabetes: a prospective cohort study. BMJ Open. 2012;2, e001730.

\section{Submit your next manuscript to BioMed Central and we will help you at every step:}

- We accept pre-submission inquiries

- Our selector tool helps you to find the most relevant journal

- We provide round the clock customer support

- Convenient online submission

- Thorough peer review

- Inclusion in PubMed and all major indexing services

- Maximum visibility for your research

Submit your manuscript at www.biomedcentral.com/submit 